\title{
Fatores de estresse e qualidade de vida de estudantes de Odontologia
}

\author{
Maria Imaculada de Queiroz Rodrigues*; Luciana Maria Arcanjo Frota**; Myrna Maria Arcanjo \\ Frota $^{* * *}$; Cinthia Nara Gadelha Teixeira****
}

* Graduanda, Curso de Odontologia, Universidade Federal do Ceará

** Mestre em Clínica Odontológica, Universidade Federal do Ceará

*** Doutoranda em Clínica Odontológica, Docente do Curso de Odontologia, Universidade Federal do Ceará, Campus Sobral

**** Doutoranda em Odontologia, Universidade Federal do Maranhão, Docente do Curso de Odontologia, Centro Universitário Christus

Recebido em 28/02/2018. Aprovado em 12/02/2019.

\begin{abstract}
RESUMO
O objetivo do estudo foi realizar uma revisão integrativa sobre os fatores de estresse e qualidade de vida de estudantes de graduação em Odontologia. Foram selecionados artigos publicados entres os anos de 2014 e 2017, publicados na íntegra, em português ou inglês. A busca de dados foi realizada nas bases de dados Scientific Eletronic Library Online (SciELO), Literatura Latino-americana e do Caribe em Ciências da Saúde (LILACS) e U.S. National Institutes of Health's National Library of Medicine (PubMed), por meio da combinação - utilizando o operador booleano "AND" - dos descritores, em português e inglês: "Qualidade de Vida/Quality of Life", "Estudantes de Odontologia/Students, Dental", "Educação Superior/Education, Higher". Foram encontrados 5.537 artigos, sendo 10 selecionados. A LILACS foi a base de dados com mais estudos selecionados (80\%) e todos os estudos foram classificados no nível IV de evidência científica. Foram identificados estudos que analisaram os níveis de depressão, ansiedade, estresse e qualidade de vida, bem como os fatores mais estressantes entre estudantes de Odontologia em universidades públicas e privadas. Concluiu-se que diversos fatores são estressantes para acadêmicos de Odontologia, entre os quais destacam-se a alta carga de trabalho durante o curso e o medo de falhar durante a graduação. Estudos que analisem a qualidade de vida destes estudantes ainda são escassos na literatura.
\end{abstract}

Descritores: Qualidade de Vida. Estudantes de Odontologia. Educação Superior. 


\section{INTRODUÇÃO}

A qualidade de vida (QV) é definida pela Organização Mundial da Saúde (OMS) "como a percepção do indivíduo de sua posição na vida, no contexto da cultura e sistemas de valores nos quais vive e em relação aos seus objetivos, expectativas, padrões e preocupações". Porém, embora tenha uma definição oficial, a QV se trata de uma construção complexa, com indicadores diversos que vão desde questões emocionais a aspectos financeiros e espirituais ${ }^{1}$.

Com o intuito de entender o complexo contexto da $\mathrm{QV}$, estudos têm sido realizados a fim de obter resultados sobre a $\mathrm{QV}$ de diversos setores da população. Em especial, a QV de estudantes durante o curso de graduação tem sido alvo de muitas pesquisas ${ }^{1,2}$.

Os cursos de graduação em Odontologia por todo o mundo são caracterizados pelo alto nível de estresse para os estudantes ${ }^{3}$. Nos primeiros anos do curso, que se configura como o período pré-clínico, há uma alta carga teórica, que inclui conceitos básicos para a prática odontológica e desenvolvimento de habilidades clínicas necessárias à atividade profissional ${ }^{2}$. Fatores associados a esse período podem afetar a QV dos estudantes, como a grande carga horária teórica, a competição acadêmica que se estabelece dentro das turmas, a consequente busca por notas cada vez mais altas, além do medo de fracassar diante de tantas exigências ${ }^{4,5}$.

Nos últimos anos de formação, além da carga teórica, os estudantes passam a frequentar as clínicas, atendendo pacientes ${ }^{6}$. Durante essa última fase da formação, os fatores associados ao estresse são a responsabilidade de cuidar de um paciente, realização de tratamentos irreversíveis, cooperação de pacientes difíceis, além das elevadas exigências de habilidades práticas ${ }^{3,6}$.

Todos esses fatores contribuem para o estresse no ambiente acadêmico odontológico, e este, por conseguinte, pode resultar em transtornos mentais, como elevados níveis de depressão, transtornos de ansiedade, exaustão emocional e eventualmente com repercussões somáticas. Todas essas consequências do estresse podem resultar em efeitos prejudiciais à QV dos estudantes, dificultando também o seu aprendizado e interferindo negativamente nos resultados $\operatorname{acadêmicos}^{1,2,5-7}$.

Dessa maneira, torna-se extremamente relevante o estudo da QV entre estudantes dos cursos de graduação em Odontologia. Trabalhos com esse enfoque contribuem na orientação de projetos voltados à promoção de saúde e sucesso dentro do espaço universitário. Tais ações proporcionarão melhor desempenho acadêmico e, por consequência, podem repercutir também em bons resultados na vida profissional dos futuros cirurgiões-dentistas ${ }^{7,8}$.

O objetivo dessa revisão integrativa foi analisar os fatores de estresse e a QV de estudantes de graduação em Odontologia em diversas instituições públicas e privadas do mundo.

\section{MÉTODOS}

O presente estudo se trata de uma revisão integrativa da literatura. A pergunta norteadora da pesquisa foi "Quais os fatores estressores e como se caracteriza a QV de estudantes de Odontologia?" Foram incluídos apenas artigos científicos publicados na íntegra, em português ou inglês, no período de 2014 a 2017, que estivessem relacionados ao tema deste trabalho.

A busca dos artigos foi realizada nas bases de dados Scientific Eletronic Library Online (SciELO), Literatura Latino-americana e do Caribe em Ciências da Saúde (LILACS) e U.S. National Institutes of Health's National Library of Medicine (PubMed), por meio da combinação - utilizando o operador booleano "AND" - dos descritores, em português e inglês: "Qualidade de Vida/Quality of Life", "Estudantes de Odontologia/Students, Dental", "Educação Superior/Education, Higher". 
Os critérios de exclusão foram: pesquisas realizadas com estudantes de pós-graduação, revisões sistemáticas e revisões de literatura, além dos trabalhos que não se adequavam ao tema proposto.

Para análise de qualidade dos estudos, foi utilizada a classificação do nível de evidência (NE): nível I - metanálise de estudos clínicos controlados e com randomização; nível II - estudo de desenho experimental; nível III - pesquisas quase-experimentais; nível IV - estudos descritivos ou com abordagem metodológica qualitativa; nível V - relatórios de casos ou relatos de experiências; nível VI - opiniões de especialistas ou com base em normas ou legislação? .

O presente estudo respeitou os aspectos éticos das pesquisas, sendo fiel a autoria das ideias, conceitos e definições presentes nos trabalhos que fizeram parte desta revisão.

\section{RESULTADOS}

Foram encontrados 5.537 trabalhos, sendo 148 na base de dados SciELO, 2.412 na LILACS e 2.977 na PubMed. Levando em consideração os critérios de inclusão e exclusão, foram selecionados 10 artigos.

A tabela 1 mostra as estratégias de busca, bases consultadas, quantidade de trabalhos encontrados e selecionados para o estudo. A base de dados LILACS teve o maior número de artigos selecionados (80\%). As combinações dos descritores "Qualidade de Vida" e "Educação Superior", em português e inglês, geraram o maior número de artigos na busca inicial. Quanto à seleção final dos artigos, as combinações dos descritores "Estudantes de Odontologia" e "Educação Superior", em português e inglês, foi a que gerou mais artigos para compor esta revisão.

Tabela 1. Estratégias de busca, bases consultadas, número de referências encontradas e selecionadas

\begin{tabular}{l|c|c|c}
\hline Estratégias de busca & $\begin{array}{c}\text { Bases } \\
\text { consultadas }\end{array}$ & $\begin{array}{c}\text { Referências } \\
\text { encontradas }\end{array}$ & $\begin{array}{c}\text { Referências } \\
\text { selecionadas }\end{array}$ \\
\hline $\begin{array}{l}\text { Qualidade de Vida/Quality of Life [Palavras] } \\
\begin{array}{l}\text { AND Estudantes de Odontologia/Students, } \\
\text { Dental [Palavras] }\end{array}\end{array}$ & SciELO & 4 & - \\
\hline & PILACS & 112 & - \\
\hline $\begin{array}{l}\text { Qualidade de Vida/ Quality of Life [Palavras] } \\
\text { AND Educação Superior/ Education, Higher }\end{array}$ & SciELO & 144 & - \\
[Palavras] & LILACS & 1.839 & - \\
\hline $\begin{array}{l}\text { Estudantes de Odontologia/ Students, Dental } \\
\text { [Palavras] AND Educação Superior/ Education, }\end{array}$ & LILACS & 452 & - \\
Higher [Palavras] & PubMed & 322 & 5 \\
\hline $\begin{array}{l}\text { Qualidade de Vida/ Quality of Life [Palavras] } \\
\text { AND Estudantes de Odontologia/Students, }\end{array}$ & SciELO & - & 2 \\
$\begin{array}{l}\text { Dental [Palavras] AND Educação Superior/ } \\
\text { Education, Higher [Palavras] }\end{array}$ & LILACS & 09 & - \\
\hline TOTAL & PubMed & 10 & - \\
\hline
\end{tabular}


Na tabela 2 estão expressos os dados da análise inicial dos artigos selecionados, tais como autor(es)/ano de publicação, país de origem da publicação, título do artigo e base de dados de origem. Dentre os artigos selecionados, 100\% foram publicados no idioma inglês. A maioria dos estudos foram publicados em 2015 (40\%) e 2017 (40\%). A Turquia foi o país com maior prevalência quanto ao país de origem do autor principal (20\%).

Tabela 2. Caracterização dos estudos selecionados quanto a autor/ano, país de afiliação do autor principal, título do artigo e bases de dados

\begin{tabular}{|c|c|c|c|}
\hline Autor/Ano & $\begin{array}{c}\text { País de } \\
\text { afiliação do } \\
\text { autor principal }\end{array}$ & Título do artigo & $\begin{array}{c}\text { Base de } \\
\text { dados }\end{array}$ \\
\hline $\begin{array}{l}\text { Mafla et al., } \\
2014\end{array}$ & Colômbia & $\begin{array}{l}\text { Burnout prevalence and correlates amongst } \\
\text { Colombian dental students: the STRESSCODE study }\end{array}$ & LILACS \\
\hline $\begin{array}{l}\text { Aboalshamat } \\
\text { et al., } 2015\end{array}$ & Arábia Saudita & $\begin{array}{l}\text { Psychological well-being status among medical and } \\
\text { dental students in Makkah, Saudi Arabia: A cross- } \\
\text { sectional study }\end{array}$ & LILACS \\
\hline $\begin{array}{l}\text { Atalayin et } \\
\text { al., } 2015\end{array}$ & Turquia & $\begin{array}{l}\text { The prevalence and consequences of burnout on a } \\
\text { group of preclinical dental students }\end{array}$ & LILACS \\
\hline $\begin{array}{l}\text { Bathla et al., } \\
2015\end{array}$ & Índia & $\begin{array}{l}\text { Evaluation of anxiety, depression and suicidal intent in } \\
\text { undergraduate dental students: A cross-sectional study }\end{array}$ & LILACS \\
\hline $\begin{array}{l}\text { Babar et al., } \\
2015\end{array}$ & Malásia & $\begin{array}{l}\text { Perceived sources of stress among Malaysian dental } \\
\text { students }\end{array}$ & PubMed \\
\hline $\begin{array}{l}\text { Burger et al., } \\
2016\end{array}$ & Alemanha & $\begin{array}{l}\text { Development of depression and deterioration in } \\
\text { quality of life in German dental medical students in } \\
\text { preclinical semesters }\end{array}$ & LILACS \\
\hline $\begin{array}{l}\text { Harris et al., } \\
2017\end{array}$ & Reino Unido & $\begin{array}{l}\text { Perceived stress and well-being among dental hygiene } \\
\text { and dental therapy students }\end{array}$ & LILACS \\
\hline $\begin{array}{l}\text { Andre et al., } \\
2017\end{array}$ & Estados Unidos & $\begin{array}{l}\text { Quality of Life Among Dental Students: A Survey } \\
\text { Study }\end{array}$ & LILACS \\
\hline $\begin{array}{l}\text { Ersan et al., } \\
2017\end{array}$ & Turquia & $\begin{array}{l}\text { Perceived sources and levels of stress, general self- } \\
\text { efficacy and coping strategies in clinical dental } \\
\text { students }\end{array}$ & LILACS \\
\hline $\begin{array}{l}\text { Hayes et al., } \\
2017\end{array}$ & Canadá & $\begin{array}{l}\text { Perceived causes of stress among a group of western } \\
\text { Canadian dental students }\end{array}$ & PubMed \\
\hline
\end{tabular}

Na tabela 3 estão descritos dados como autor(es)/ano de publicação, objetivo dos trabalhos e nível evidência dos estudos, segundo a proposta da pesquisa. Todos os estudos foram classificados no nível IV de evidência (100\%), tendo em vista que todos foram estudos descritivos (não experimentais), relatando as observações feitas, por meio de questionários, sobre a QV de estudantes de diversas escolas de Odontologia no mundo. 
Tabela 3. Autor(es), ano de publicação, objetivos e nível de evidência dos estudos selecionados

\begin{tabular}{llc}
\hline Autor/Ano & \multicolumn{1}{c}{ Objetivos do estudo } & $\begin{array}{c}\text { Nível de } \\
\text { evidência }\end{array}$ \\
\hline $\begin{array}{l}\text { Mafla } \text { et al., } \\
2014\end{array}$ & $\begin{array}{l}\text { Determinar a prevalência de Burnout entre uma amostra de } \\
\text { estudantes de Odontologia colombianos e investigar suas } \\
\text { correlações psicossociais e educacionais. }\end{array}$ & IV \\
\hline $\begin{array}{l}\text { Aboalshama } \\
\text { t } \text { et al., }\end{array}$ & $\begin{array}{l}\text { Avaliar o bem-estar psicológico entre os estudantes de Medicina } \\
\text { e Odontologia na Arábia Saudita, identificar os grupos de alto } \\
\text { risco e avaliar a associação entre o bem-estar psicológico e o } \\
\text { desempenho acadêmico. }\end{array}$ & IV \\
\hline $\begin{array}{l}\text { Atalayin } \text { et } \\
\text { al., } 2015\end{array}$ & $\begin{array}{l}\text { Investigar a prevalência de Burnout entre um grupo de estudantes } \\
\text { de Odontologia, da etapa pré-clínica, na Turquia. }\end{array}$ & IV \\
$\begin{array}{l}\text { Bathla } \text { et } \\
\text { al., } 2015\end{array}$ & $\begin{array}{l}\text { Avaliar ansiedade, depressão e intenção suicida nos estudantes } \\
\text { de graduação em Odontologia e descobrir as diversas áreas do } \\
\text { estresse. }\end{array}$ & IV \\
\hline $\begin{array}{l}\text { Babar } \text { et al., } \\
2015\end{array}$ & $\begin{array}{l}\text { Identificar os níveis de estresse e explorar o impacto do ano de } \\
\text { estudo e gênero dos alunos sobre as fontes de estresse percebidas } \\
\text { entre estudantes de Odontologia da Malásia. }\end{array}$ & IV \\
\hline $\begin{array}{l}\text { Burger } \text { et } \\
\text { al., } 2016\end{array}$ & $\begin{array}{l}\text { Avaliar a prevalência de Burnout em estudantes de uma escola } \\
\text { de Odontologia. }\end{array}$ & IV \\
\hline $\begin{array}{l}\text { Harris } \text { et al., } \\
2017\end{array}$ & $\begin{array}{l}\text { Explorar a percepção de estresse e bem-estar dos estudantes de } \\
\text { Odontologia durante a graduação. }\end{array}$ & IV \\
\hline $\begin{array}{l}\text { Andre } \text { et al., } \\
2017\end{array}$ & $\begin{array}{l}\text { Avaliar a qualidade de vida dos estudantes de Odontologia em } \\
\text { uma faculdade de Odontologia dos EUA }\end{array}$ & IV \\
\hline $\begin{array}{l}\text { Identificar fontes de estresse entre os estudantes clínicos e avaliar } \\
\text { os níveis percebidos de estresse, auto eficácia geral e estratégias } \\
\text { de enfrentamento efetivas dos alunos em faculdade privada de } \\
\text { Odontologia. }\end{array}$ & IV \\
\hline $\begin{array}{l}\text { Hayes } \text { et al., } \\
\text { Universinar como os estudantes da Faculdade de Odontologia da }\end{array}$ & IV \\
\hline
\end{tabular}

Alguns estudos (30\%) avaliaram a prevalência de Burnout entre acadêmicos de Odontologia, que se caracteriza como um estado de esgotamento ou desgaste físico e mental ${ }^{2,7,10}$.

Em estudo realizado com um grupo de estudantes de Odontologia colombianos de instituições privadas e públicas, aferiu-se que os maiores níveis de esgotamento físico e mental se encontravam entre estudantes de instituições públicas, que estudavam em classes muito numerosas, bem como entre estudantes mais velhos, casados e que não tinham a Odontologia como primeira escolha de carreira. Foi constatado que o ambiente educacional influenciou nos níveis de Burnout entre os estudantes, com destaque para o estresse em virtude da alta carga de trabalho durante o curso ${ }^{10}$.

Em estudo realizado na Turquia, com estudantes da etapa pré-clínica do curso de Odontologia de uma universidade pública, os resultados mostraram que cerca de $22 \%$ dos estudantes analisados apresentavam altos níveis de Burnout, e a alta carga de trabalho esteve relacionada ao desenvolvimento desse quadro. Os níveis mais elevados estavam entre alunos no terceiro ano do curso e estudantes do sexo 
feminino. Ainda, dentre os estudantes com maiores níveis de exaustão física e mental, foram observados os menores níveis de satisfação acadêmica. Dentre os estudantes vivendo distantes da família, foram identificados os menores níveis de performance acadêmica ${ }^{2}$.

Ainda, em estudo realizado na Alemanha entre estudantes de Odontologia de uma universidade pública, observou-se que no $5^{\circ}$ período houve prevalência de indivíduos com níveis de depressão clinicamente expressos. Além disso, foi observada uma contínua diminuição da QV mental dos estudantes paralela à progressão da graduação, evidenciando a deterioração da saúde mental desses alunos, que se relaciona aos níveis de depressão observados ${ }^{7}$. Os autores concordam que o desenvolvimento de currículos que consigam suprir as necessidades individuais dos alunos, conscientiza-los sobre como lidar com o estresse e melhorar a comunicação com os estudantes pode diminuir os níveis de Burnout na graduação ${ }^{27,10}$.

Estudo realizado na Malásia avaliou os níveis e fontes de estresse entre estudantes de universidades públicas e privadas de Odontologia do país. Observou-se que os acadêmicos de universidades públicas e do sexo feminino apresentaram maiores níveis de estresse. Os maiores fatores estressantes estavam relacionados às questões acadêmicas, como o medo de falhar durante a graduação e os resultados dos testes aplicados durante o curso. Ainda, nos anos iniciais de graduação, um fator de estresse foi o medo do desemprego após o término da formação. Os autores destacam a necessidade do desenvolvimento de programas de gerenciamento do estresse durante a graduação ${ }^{8}$.

Outra pesquisa, realizada com estudantes de Medicina e Odontologia de uma universidade pública no estágio pré-clínico, revelou altos níveis de depressão, ansiedade e estresse entre os alunos. No curso de Odontologia, os estudantes ficaram mais deprimidos no terceiro ano e mais estressados no segundo ano. Além disso, os alunos do sexo masculino apresentaram maior sofrimento psicológico do que estudantes do sexo feminino. Os autores afirmam ser necessário o emprego de estratégias para a redução desses níveis de estresse $^{4}$.

O estresse e bem-estar de estudantes de Odontologia foi avaliado em uma instituição pública no Reino Unido. Fatores como a prática clínica, o medo de falhar e atender aos requisitos para ser um bom clínico, bem como os trabalhos acadêmicos foram os maiores geradores de estresse. Nesses alunos, os níveis de depressão, ansiedade e estresse estavam considerados dentro da faixa de normalidade, e esses se autoavaliaram como indivíduos de funcionamento positivo ${ }^{5}$.

Outro estudo objetivou avaliar os níveis de ansiedade, depressão e intenção suicida entre estudantes de Odontologia de uma instituição privada. Os maiores níveis de ansiedade e depressão foram relatados pelos alunos do primeiro e último ano de graduação. A intenção suicida foi relatada por alunos de todos os anos do curso. Os fatores mais associados ao estresse foram longas horas de ensino, alta carga de trabalho, elevada frequência de aplicação de testes, competição entre os colegas de turma e medo de falhar. Além disso, o não interesse pela profissão também foi um fator de estresse. Os autores ressaltam a necessidade de mudanças no sistema educacional vigente $\mathrm{e}$ enfatizam a importância de desenvolver-se estratégias de gerenciamento do estresse na graduação ${ }^{6}$.

Nos Estados Unidos avaliou-se a QV de estudantes de uma faculdade privada de Odontologia. Nesse estudo, os alunos classificaram sua QV como boa. No domínio Relações Sociais, as mulheres demonstraram melhor QV, em relação aos estudantes do sexo masculino. Ainda, os estudantes solteiros e mais velhos tinham pior QV que estudantes casados e mais novos, respectivamente. Além disso, os escores de QV no 
domínio Psicológico foram menores para os alunos do terceiro ano de curso, em relação ao primeiro ano, domínio esse que trata dos níveis de satisfação com a vida, acreditar em si mesmo, autoestima e sentimentos negativos. Os autores destacam que oferecer apoio a esses estudantes é essencial não só para promover QV entre eles, mas também melhorar o atendimento aos pacientes e a profissão como um todo ${ }^{1}$.

Estudo realizado em uma escola privada de Odontologia na Turquia, afim de avaliar fontes e níveis de estresses entre estudantes, constatou que o último ano de graduação foi considerado o mais estressante e os estudantes do sexo feminino tiveram os níveis mais altos de estresse. A alta carga de trabalho e o treinamento clínico foram as maiores fontes estressoras. Reduzir a sobrecarga do currículo e implementar estratégias de gerenciamento do estresse, principalmente para estudantes mais avançados no curso podem ser medidas eficazes, segundo os autores ${ }^{3}$.

O último estudo desta revisão integrativa foi realizado com estudantes de Odontologia de uma universidade privada do Canadá, afim de avaliar como estes percebem o estresse durante a graduação. Foi observado que as estudantes do sexo feminino tinham níveis mais elevados de estresse que o sexo masculino. Estudar fora de casa, mudança dos estudos pré-clínicos para os clínicos, preocupação com os requisitos para ser um bom clínico, além da alta carga de trabalho nas clínicas e elevadas dívidas com as mensalidades do curso foram relatados como fatores estressantes. Os autores afirmam que treinar professores para gerenciamento do estresse dos alunos apresenta-se como uma estratégia viável de manejo dessa problemática $^{11}$.

\section{DISCUSSÃO}

$\mathrm{Na}$ busca foi encontrado um número expressivo de publicações. Entretanto, o reduzido número de artigos selecionados para fazer parte desta revisão integrativa se deu pela vasta quantidade de avaliações de métodos de ensino em cursos da saúde, em especial da área médica, principalmente quanto utilizados os descritores "Students, Dental" e "Education, Higher" em associação. Ainda, grande parte dos estudos se tratava de análises da QV de pacientes realizadas por estudantes de graduação. Usando as estratégias de busca e critérios de inclusão e exclusão já mencionados, não foram selecionados estudos brasileiros, o que mostra escassez de pesquisas sobre o tema no país.

Grande parte dos estudos analisou os níveis e fatores de estresse entre estudantes de Odontologia, fatores estes que afetam a QV e interferem também no desempenho acadêmico ${ }^{2-}$ 8,10,11. Apenas um estudo avaliou diretamente a QV de estudantes de graduação em Odontologia, evidenciando a escassez de estudos com essa temática na literatura ${ }^{1}$.

Foi observado que os estudantes de instituições públicas tiveram maiores índices de estresse e esgotamento físico e mental ${ }^{8,10}$. Tais resultados estão associados principalmente ao nível de exigência de estudantes em instituições públicas $^{8,10}$. Já entre estudantes de universidades privadas, foi observado que os custos com as mensalidades são um importante fator de estresse ${ }^{11}$.

A maioria dos artigos aponta que as estudantes do sexo feminino demonstram maiores níveis de estresse que os do sexo masculino, tanto nas universidades públicas, quanto nas privadas ${ }^{2-}$ 4,8,11 . Contudo, em um dos estudos, realizado em uma instituição privada, as mulheres tenham apresentado melhores escores de QV do que os homens $^{1}$. Isso pode estar associado à maior expressividade de sentimentos pelas mulheres, evidenciando menores escores de QV, enquanto os homens respondem de forma menos expressiva às suas preocupações, apresentando melhor autoavaliação da $\mathrm{QV}^{3,8}$.

O terceiro ano de graduação foi o período 
mais estressante entre os estudantes de Odontologia, principalmente entre aqueles que estudam em universidades públicas ${ }^{1,2,4,7}$. No terceiro ano do curso há a evidenciação do acúmulo de estresse dos anos anteriores, gerando níveis mais elevados nesse período. Além disso, nessa etapa da graduação, há uma maior carga de trabalho e aumento da responsabilidade associada à fase clínica do curso, em que há o atendimento direto aos pacientes ${ }^{1,2,4}$.

O medo de falhar durante a graduação foi muito comum nos estudos como fator de estresse entre estudantes, tanto em instituições públicas quanto privadas ${ }^{5,6,8}$. Finalmente, um dos fatores de estresse que mais afetaram a QV dos estudantes de Odontologia, principalmente em instituições privadas, foi a elevada carga de trabalho durante os anos de graduação, principalmente durante os anos em que há atendimento clínico ao paciente $3,6,10,11$. Isso se deve às várias avaliações de conhecimento aplicadas durante o curso, responsabilidades adquiridas ao lidar diretamente com o paciente, curto tempo livre para exercer atividades sociais $\mathrm{e}$ exigências de variados conhecimentos teóricos e clínicos necessários à prática odontológica ${ }^{6}$.

\section{CONCLUSÃO}

Os estudos selecionados demonstraram que o curso de graduação em Odontologia apresenta diversos fatores de estresse, tantos nas instituições públicas de ensino quanto nas privadas, e estes parecem afetar mais as alunas. Dentre esses fatores, ganham destaque o ano de graduação, a alta carga de trabalho durante o curso, bem como o medo de falhar durante a graduação. Tais fatores de estresse afetam a QV, levando ao desenvolvimento de diversos distúrbios mentais, e por consequência acabam afetando também seu desempenho durante o curso.

\section{ABSTRACT}

Stress factors and quality of life of Dental

\section{students}

The objective of the study was to perform an integrative review on the stress factors and quality of life of undergraduate Dental students. Articles published between the years 2014 and 2017, published in full, in Portuguese or English, were selected. The data search was carried out in the databases Scientific Eletronic Library Online (SciELO), Latin American and Caribbean Literature in Health Sciences (LILACS) and U.S. National Institutes of Health's National Library of Medicine (PubMed), through the combination - using the Boolean operator "AND" - of the descriptors, in Portuguese and English: "Qualidade de Vida/Quality of Life", "Estudantes de Odontologia/Students, Dental", "Educação Superior/Education, Higher". A total of 5.537 articles were found, of which 10 were selected. LILACS was the database with more studies selected $(80 \%)$ and all studies were classified in level IV of scientific evidence. Studies that analyzed the levels of depression, anxiety, stress and quality of life were identified, as well as the most stressful factors among students of Dentistry in public and private universities. It was concluded that several factors are stressful for Dental scholars, among them the high workload during the course and the fear of failing during graduation. Studies that analyze the quality of life of these students are still scarce in the literature.

Descriptors: Quality of Life. Students, Dental. Education, Higher.

\section{REFERÊNCIAS}

1. Andre A, Pierre GC, McAndrew M. Quality of Life Among Dental Students: A Survey Study. J Dent Educ. 2017;81(10):1164-70.

2. Atalayin $\mathrm{C}$, Balkis $\mathrm{M}$, Tezel $\mathrm{H}$, Onal B, Kayrak G. The prevalence and consequences of burnout on a group of preclinical dental students. Eur J Dent. 2015;9(3):356-63.

3. Ersan N, Fisekçioglu E, Dölekoglu S, Oktay I; Ilgüy D. Perceived sources and levels of stress, general self-efficacy and coping 
strategies in clinical dental students. Psychol Health Med. 2017;22(10):1175-85.

4. Aboalshamat K, Hou XY, Strodl E. Psychological well-being status among medical and dental students in Makkah, Saudi Arabia: a cross-sectional study. Med Teach. 2015;37(Suppl 3):S75-81.

5. Harris M, Wilson JC, Holmes S, Radford DR. Perceived stress and well-being among dental hygiene and dental therapy students. Br Dent J. 2017;222(2):101-6.

6. Bathla M, Singh M, Kulhara P, Chandna S, Aneja J. Evaluation of anxiety, depression and suicidal intent in undergraduate dental students: A cross-sectional study. Contemp Clin Dent. 2015;6(2):215-22.

7. Burger PHM, Neumann C, Ropohl A, Paulsen F, Scholz M. Development of depression and deterioration in quality of life in German dental medical students in preclinical semesters. Ann Anat. 2016;208:183-6.

8. Babar MG, Hasan SS, Ooi YJ, Ahmed SI, Wong PS, Ahmad SF, et al. Perceived sources of stress among Malaysian dental students. Int J Med Educ. 2015;5:56-61.
9. Stetler CB, Brunell M, Giuliano KK, Morsi D, Prince L, Newell-Stokes V. Evidencebased practice and the role of nursing leadership. J Nurs Adm. 1998;28(7-8):4553.

10. Mafla AC, Villa-Torres L, Polychronopoulou A, Polanco H, Moreno-Juvinao V, ParraGalvis D, et al. Burnout prevalence and correlates amongst Colombian dental students: the STRESSCODE study. Eur J Dent Educ. 2015;19(4):242-50.

11. Hayes A, Hoover JN, Karunanayake CP, Uswak GS. Perceived causes of stress among a group of western Canadian dental students. BMC Research Notes. 2017;10(1):714.

\section{Correspondência para:}

Maria Imaculada de Queiroz Rodrigues e-mail: imaculadaqueirozr1997@ gmail.com

Faculdade de Farmácia, Odontologia e Enfermagem, Universidade Federal do Ceará Rua Monsenhor Furtado, S/N, Rodolfo Teófilo, 60430-355 Fortaleza/CE 